University of Wollongong

Research Online

Faculty of Social Sciences - Papers (Archive) Faculty of Arts, Social Sciences \& Humanities

2015

Change magnitude does not guide attention in an object change detection task

Simone Favelle

University of Wollongong, skeane@uow.edu.au

Stephen Palmisano

University of Wollongong, stephenp@uow.edu.au

Follow this and additional works at: https://ro.uow.edu.au/sspapers

Part of the Education Commons, and the Social and Behavioral Sciences Commons

Research Online is the open access institutional repository for the University of Wollongong. For further information contact the UOW Library: research-pubs@uow.edu.au 


\title{
Change magnitude does not guide attention in an object change detection task
}

\author{
Abstract \\ Investigations of change detection consistently reveal an effect of change magnitude: changes involving \\ more object parts are detected more easily than those involving fewer parts. Whether large changes \\ improve detection by providing stronger preattentive signals to the change location is subject to debate. \\ We report a cued object change detection experiment that tested this hypothesis while controlling for \\ stimulus familiarity, semantic knowledge, and change type (addition versus deletion). We found strong \\ magnitude effects regardless of whether trials were validly or invalidly cued. The size of the cueing \\ effects, which were exhibited for all the change magnitudes examined, did not decrease with the number \\ of parts changing. These findings provide little support for a preattentive guidance hypothesis and \\ instead support the thesis that change detection requires attention.

\section{Keywords} \\ does, detection, not, task, guide, attention, object, change, magnitude \\ Disciplines \\ Education | Social and Behavioral Sciences \\ Publication Details \\ Favelle, S. K. \& Palmisano, S. (2015). Change magnitude does not guide attention in an object change \\ detection task. Perception, 44 (1), 93-99.
}




\title{
SHORT REPORT \\ Change magnitude does not guide attention in an object change detection task
}

\author{
Simone K Favelle, Stephen Palmisano \\ School of Psychology, University of Wollongong, Northfields Ave, Wollongong, NSW 2522, \\ Australia; e-mail: simone_favelle@uow.edu.au \\ Received 10 February 2014, in revised form 19 November 2014, published online 13 January 2015
}

\begin{abstract}
Investigations of change detection consistently reveal an effect of change magnitude: changes involving more object parts are detected more easily than those involving fewer parts. Whether large changes improve detection by providing stronger preattentive signals to the change location is subject to debate. We report a cued object change detection experiment that tested this hypothesis while controlling for stimulus familiarity, semantic knowledge, and change type (addition versus deletion). We found strong magnitude effects regardless of whether trials were validly or invalidly cued. The size of the cueing effects, which were exhibited for all the change magnitudes examined, did not decrease with the number of parts changing. These findings provide little support for a preattentive guidance hypothesis and instead support the thesis that change detection requires attention.
\end{abstract}

Keywords: visual attention, spatial cueing, change detection

\section{Background}

Visual change detection is an important skill and one that has been investigated extensively (see Rensink, 2002; Simons \& Rensink, 2005). It is now well established that focused attention plays an important role in visual change detection. For example, Rensink, O'Regan, and Clark (1997) showed that changes which are physically or semantically central to a scene are better detected. Similarly, Scholl (2000) showed that changes made to arrays of common objects in a flicker task are easier to detect when they are accompanied by a nonverbal exogenous cue (such as a colour singleton). Most change detection findings can be accounted for by the thesis that attention is necessary to see change (Rensink, 2002). As intuitive as this seems, however, the necessity of attention for change detection remains a controversial proposition. In support of the notion that change detection processes might operate outside of attention, Fernandez-Duque and Thornton $(2000,2003)$ demonstrated that observers could locate and identify changes at better than chance levels, regardless of their awareness of the change occurring. They argued that, even when we are unaware that a change has occurred, implicit perception of change can still influence our change detection behaviour. Along similar lines, several other researchers have argued that unattended information can play a functional role in change detection (eg Laloyaux, Destrebecqz, \& Cleeremans, 2006; Smilek, Eastwood, \& Merikle, 2000), but this point is in dispute (Mitroff, Simons, \& Franconeri, 2002).

While the issue of whether change detection may be implicit is difficult to resolve, the question of whether preattentive processing of change can direct focal attention may be less difficult to address. Smilek et al. (2000), for example, examined whether unattended changes could direct or shift the focus of attention to the location of the change in a change detection task. They reported shallower search slopes in a flicker version of a standard visual search task for stimulus conditions in which larger numbers of features changed compared with smaller numbers of features. Smilek and colleagues argued that this finding arose because larger numbers of features changing provided a greater preattentive signal to the location of change. That is, they claimed that unattended changes guided focal attention and that the preattentive processing of larger changes directed the focus of attention more efficiently to the location of change. 
Smilek et al.'s proposal stands in stark contrast to the proposition that attention is necessary for change detection. However, their results do not rule out the possibility that attention was first allocated to the location of the change, with the processing benefits of larger magnitude changes coming afterwards. Accordingly, Stolz and Jolicoeur (2004) retested Smilek et al.'s (2000) 'preattentive guidance hypothesis' with a spatial cuing task to manipulate attention more directly. They argued that if the benefits of larger numbers of features changing was preattentive in origin, then change magnitude should have no effect on detection when the change location is validly cued (since any preattentive signals to the change location would be redundant in this situation, as they would come after attention had already been directed to this location by the explicit cue). However, contrary to this preattentive guidance hypothesis, spatial cueing was found to provide equivalent improvements to the detection of both small and large number part changes. Thus, they invoked a serial processing argument to account for their results and those of Smilek et al., in which attention was first oriented to a target location and then after this larger magnitude changes were detected with a higher probability in the comparison process. ${ }^{(1)}$

In the current experiment we reexamined this serial processing account of change detection controlling for potential stimulus confounds present in the Smilek et al. (2000) and Stolz and Jolicoeur (2004) studies. Specifically, both studies used highly familiar alphanumeric characters as stimuli (letters and digits) with changes consisting of between 2 and 5 line features being added or deleted. Thus, there were two types of change involved: a change in the existence of a part and a change to the semantic identity of an item. It is difficult to compare different types of changes (see Rensink, 2002). While it is not clear how or whether these different types of change might interact with each other or with the magnitude of change in a detection task, a more quantitative measure of change would allow us to discount at least some possible confounds.

With regards to the existence of a part, whether the change is an onset or offset (ie an object part addition or deletion) may influence patterns of performance as additions to a display of simple shapes are known to have significant reaction time (RT) and accuracy advantages in a change detection task (Cole, Kentridge, Gellatly, \& Heywood, 2003). It is also possible that magnitude effects may be more pronounced for one change type over the other; however, in both the Smilek et al. and Stolz and Jolicouer studies additions and deletions were treated as equivalent.

Surprisingly often overlooked in change detection research is the importance of semantic knowledge. On the one hand, there is the problem of how the magnitudes of semantic changes might be quantified (eg how does the change from vowel to consonant compare with that from letter to digit?). On the other hand, there are findings which suggest that (a) object familiarity influences change detection performance (Williams \& Simons, 2000) and (b) verbal strategies may assist change detection with familiar object stimuli (eg Simons, 1996). Murphy and Andalis (2013) recently demonstrated that unattended semantic information (masked object labels) can facilitate change detection and identification in real-world photographs of scenes. Further, they found a deleted object advantage (over addition) in both detection and identification that they attributed to the use of a verbal encoding strategy (also see Mondy \& Coltheart, 2000). According to these authors, participants made a verbal list of objects in the first scene and this list was 'checked' when the second scene was viewed. Because the deleted object was on the list, it could therefore be used as a point of comparison, making detection easier than for additions where all the items on the list were present in the second scene.

(1) Also consistent with this serial processing account, Favelle, Palmisano, Burke, and Hayward (2006) found an advantage for detecting configuration-based part changes in a single three-dimensional (3-D) object which occurred only after the allocation of attention. 
In the current study we asked participants to detect the location of change in a spatially cued one-shot object change detection task (see figure 1). We wished to examine both the role of additions and deletions and the role of change magnitude on change detection performance when minimal semantic information was available. Accordingly, we briefly displayed scenes consisting of novel, 3-D objects, with 1-3 parts being added or deleted to one of these objects between frames 1 and 2 (cf 4 s used in Murphy \& Andalis, 2013). Not only does the use of novel objects and brief initial display times render the use of a verbal strategy unlikely, but it also results in a cleaner measure of the magnitude of change. The use of novel objects can also be used to reduce any facilitation effects of prior schemas and knowledge structures (ie on the identification of the change once it has been detected; Agostinelli, Sherman, Fazio, \& Hearst, 1986).

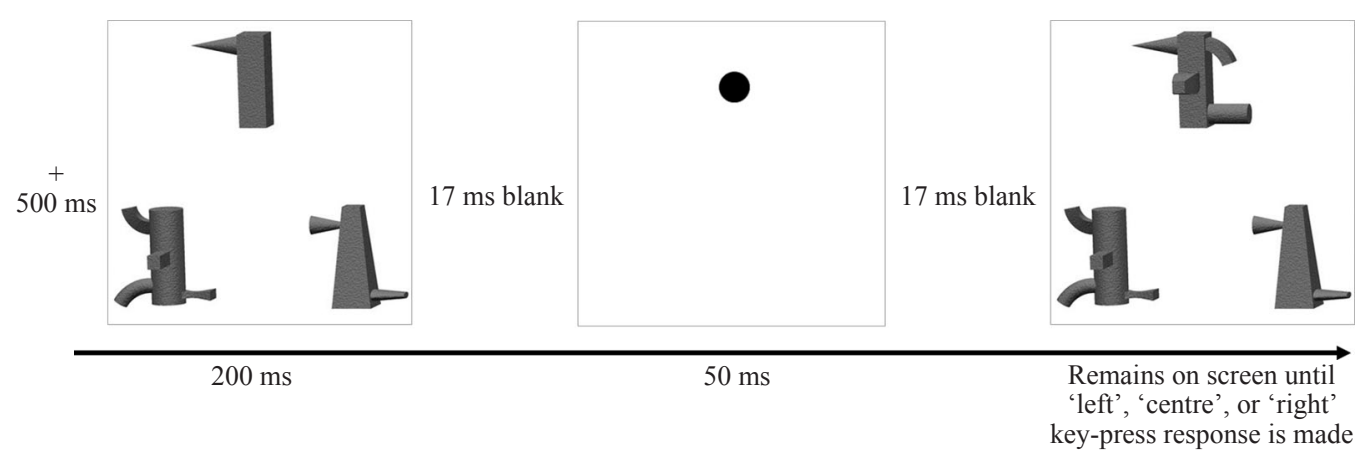

Figure 1. An example sequence of events in a validly cued, three-part addition change detection trial.

\section{Results}

\subsection{RT data analysis}

RT data for trials with a correct response were subjected to a 2 (cueing: valid or invalid) $\times 2$ (change type: addition or deletion) $\times 3$ (change magnitude: one, two, or three parts) repeatedmeasures ANOVA. As seen in figure 2 , we found strong main effects of cueing $\left(F_{1,27}=138.5\right.$, $\left.p<0.001, \eta_{\mathrm{p}}^{2}=0.84\right)$ and of change magnitude $\left(F_{2,54}=41.3, p<0.001, \eta_{\mathrm{p}}^{2}=0.59\right)$. There was no main effect of change type; it did not interact with magnitude (both $F \mathrm{~s}<1.2$ ). The three-way interaction was marginal ( $p=0.05$ ) and may be due to the cueing effect for one-part deletions being smaller than one-part additions. Change type influenced performance only in that cueing effects in RT were larger overall for additions than deletions $\left(F_{1,27}=13.9, p=0.001, \eta_{\mathrm{p}}^{2}=0.34\right)$. Critically, there was a clear interaction between cueing and change magnitude $\left(F_{2,57}=9.7\right.$, $\left.p=0.004, \eta_{\mathrm{p}}^{2}=0.26\right)$; however, this was in the opposite direction to the preattentive guidance predictions of Smilek and colleagues (2000) and consolidated a slight trend found by Stolz and Jolicoeur (2004). In contrast to the proposal that large changes are better at guiding the focus of attention, we found a significantly greater cueing effect for three-part $(153 \mathrm{~ms})$ and two-part changes $(141 \mathrm{~ms})$ than for one-part changes $(85 \mathrm{~ms}$; both $p \mathrm{~s}<0.015)$. These results suggest additive RT effects of cueing and change magnitude on the change detection. In this experiment larger magnitude changes made to the novel objects were better detected than smaller magnitude changes (regardless of the cueing). Thus, it appears that the familiarity of the stimuli used in previous studies may have attenuated the effects of change magnitude.

\subsection{Inverse efficiency score analysis}

Performance accuracy in this experiment ranged from generally very good in validly cued trials to a floor effect where detection of one-part invalidly cued deletions was not significantly different from that expected by chance $\left(t_{27}=1.7, p=0.1\right) .{ }^{(2)}$ Because of the variability in

(2) Note that the average error rate for all of the uncued conditions was $28.8 \%$ or lower, demonstrating that the change detection task itself was not too easy or too hard without explicit cueing. 
error rate across conditions, an inverse efficiency score (IES), which is a combined measure of RT/accuracy, was calculated (see Akhtar \& Enns, 1989; Townsend \& Ashby, 1983). ${ }^{(3)}$ As can be seen in figure 2, there were clear change magnitude and cueing effects in the IES data. While there was a larger cueing effect for one-part changes (likely confounded by floor effects in these conditions), the size of the cueing effect was similar for two-part and threepart changes (note that Stolz \& Jolicoeur, 2004, found equivalent-sized cueing effects across all their magnitudes of change).

Results of a 2 (cueing) $\times 2$ (change type) $\times 3$ (change magnitude) repeated-measures ANOVA also showed significant main effects in the IES data, with greater inverse efficiency for invalid cues than valid cues $\left(F_{1,27}=96.3, p<0.001, \eta_{\mathrm{p}}^{2}=0.78\right)$, decreasing inverse efficiency with increasing numbers of parts changing $\left(F_{2,54}=146.3, p<0.001, \eta_{\mathrm{p}}^{2}=0.84\right)$, and greater inverse efficiency for deletions than additions $\left(F_{1,27}=64.2, p<0.001, \eta_{\mathrm{p}}^{2}=0.70\right)$.
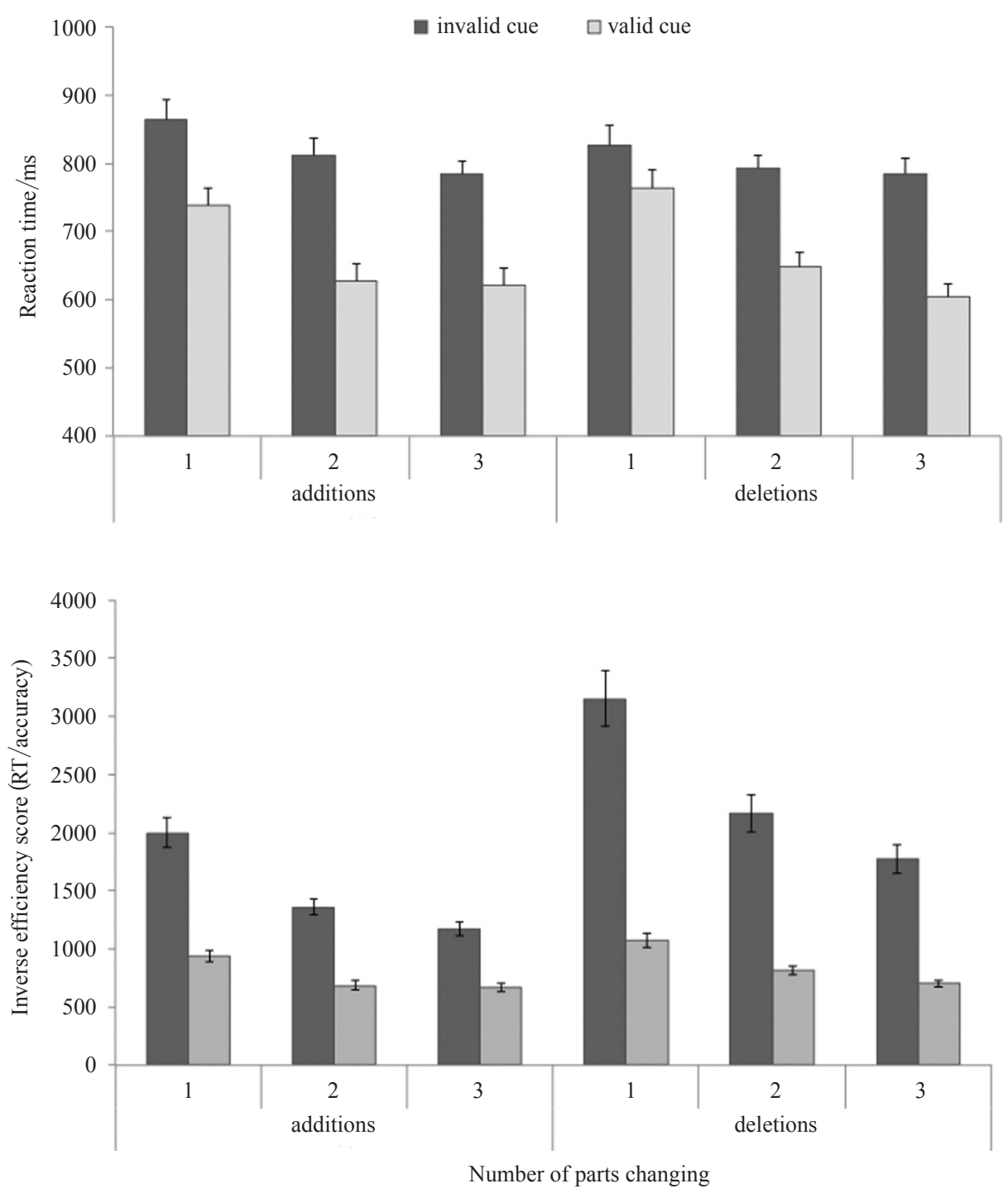

Figure 2. Mean reaction times for correct responses (top panel) and inverse efficiency scores (bottom panel) for validly and invalidly cued trials as a function of the magnitude of change (number of parts) and change type (addition and deletion). The error bars represent 1 SEM.

(3) Thank you to an anonymous reviewer for this suggestion. 
A significant interaction between cueing and change type suggested larger cueing effects for deletions than additions $\left(F_{2,54}=33.4, p<0.001, \eta_{\mathrm{p}}^{2}=0.55\right)$. Since this was opposite to the pattern found in RT, it was likely to reflect a speed-accuracy trade-off and floor effect for one-part deletions. There was a significant interaction between change type and magnitude $\left(F_{2,54}=6.9, p=0.014, \eta_{\mathrm{p}}^{2}=0.20\right)$, which, again, appeared based on the floor effect for onepart deletions creating a steeper magnitude effect for deletions over additions. There was also a significant interaction between cueing and magnitude $\left(F_{2,54}=28.6, p<0.001, \eta_{\mathrm{p}}^{2}=0.51\right)$. Bonferroni-adjusted a posteriori pairwise comparisons showed that: (i) inverse efficiency was significantly higher for invalidly, as opposed to validly, cued changes at each of the three levels of magnitude (all $p$ s $<0.001$ ); and (ii) within each level of cueing, inverse efficiency was significantly lower for three-part changes than two-part changes, and lower for two-part changes than one-part changes (all $p \mathrm{~s}<0.05)$. There was no three-way interaction $(F=3.0, p=0.07)$.

\section{Discussion}

The present experiment showed clear cueing and magnitude effects in both RT and IES, demonstrating that the spatial cues directed attention and the number of parts changing affected task difficulty. The data did not support the preattentive guidance hypothesis for visual change detection. If large changes provide stronger preattentive signals which are able to direct focal attention, then one should expect: (i) little to no effect of change magnitude on validly cued trials (since the preattentive signal would render the valid cue redundant), and (ii) costs for invalid cues (relative to valid cues) to be less for large feature changes than for smaller feature changes (since the preattentive signal would be in conflict with the invalid cue). However, larger magnitude changes did not disproportionately improve either the speed or efficiency of detection in invalidly cued conditions. Thus, large magnitude changes did not appear to provide extra signals to change locations (or if any such signals were present, they did not appear to be able to attenuate the experimental cueing effects). In fact, the RT discrepancy between valid and invalid cues actually increased with the change magnitude. Valid cues improved detection performance (in terms of both RT and IES) regardless of the magnitude of change. These results do not rule out the possibility that some form of change detection may occur without attention, but they do appear to rule out any primary role they might have in directing focal attention. Overall, our results provide support for the serial processing argument in which the effect of change magnitude exerts its influence on detection performance only after attention has been allocated (Favelle et al., 2006; Stolz \& Jolicoeur, 2004).

\section{Experimental procedure}

\subsection{Participants}

A total of thirty undergraduate students (twenty-one female) were tested individually and received course credit for their participation. All had normal or corrected-to-normal vision. Data from two participants were not included in analyses due to performance above 3 SDs from the mean (IES) in at least one condition. The final sample size was twenty eight (nineteen female).

\subsection{Stimuli}

Stimuli were rendered images of 3-D novel objects. Each object was of a similar overall size (subtending approximately $5.7 \times 4.3 \mathrm{deg}$ ) and was composed of a main body with one, two, three, or four parts attached to the body at nine possible positions (see figure 1). There were six different object bodies, and each had 15 different versions in which one, two, three, or four parts were visible, giving a total of 90 different object exemplars used in the current experiment. The cue was a small black filled circle (50 pixel diameter, subtending approximately $1.4 \times 1.4 \mathrm{deg}$ ). 
The screen background was white. The experiment was controlled by RSVP software (http:// www.tarrlab.org) on Macintosh computers with 17" Macintosh CRT monitors $(1024 \times 768$ pixels).

\subsection{Procedure}

Participants were given both verbal and written (on the computer screen) instructions on how to complete the task. After reading the instructions, participants completed 18 practice trials to familiarise them with the task. Following the practice trials, participants were given a chance to ask any questions about the procedure, should they have any, before continuing with the experiment. The experiment consisted of 648 randomly ordered trials with self-paced breaks every 54 trials. Each trial began with a fixation cross appearing for $500 \mathrm{~ms}$ at the centre of the screen, followed by a triangular array of three objects presented for $200 \mathrm{~ms}$ (see figure 1). In cued trials this first object array was followed by a $17 \mathrm{~ms}$ blank interval and then a cue (black circle) for $50 \mathrm{~ms}$ then another $17 \mathrm{~ms}$ blank interval. In no-cue trials, the first object array was followed by a $84 \mathrm{~ms}$ blank interval. The second object array was then presented and remained on screen for $5000 \mathrm{~ms}^{(4)}$ or until participants made a key-press response to indicate the location of the changed object (three keys on a computer keyboard were labelled 'left', 'centre', and 'right'). Feedback was provided in the form of an audible beep to an incorrect response. There was a $500 \mathrm{~ms}$ interval before the next trial began.

Only one object in any of the three locations in the array would be involved in a change in any given trial. Changes could consist of the addition or deletion (change type) of one, two, or three parts of an object split equally among all experimental trials. The 'body' of the object was different for all three objects and never changed across the two displays in a trial. There were three cue conditions also split equally among all experimental trials: (i) a valid cue to the location of change, (ii) an invalid cue to the location of change, and (iii) no cue. The maximum number of parts visible on all three objects (not including their bodies) in each trial ranged from 6 to 12. To minimise potential distractor effects of nonchanging parts, the maximum number of visible parts (which was seen on either frame 1 or 2 depending on the trial) was approximately equated for each of the 18 conditions ${ }^{(5)}$ tested. Participants were informed that when present, the cue would not reliably indicate the location of the change. The 'no-cue' condition was included to encourage participants to focus on the change detection task and to deemphasise the cue when present. We note that, as would be expected, the mean RT for the uncued trials fell between the valid and invalid cue conditions. That is, the uncued trials had faster RT than invalidly cued trials and slower RT than validly cued trials in all change type and magnitude conditions. The uncued trial data were not included in any statistical analyses.

Statement of ethics. This research was conducted in accordance with the University of Wollongong's Human Research Ethics Committee.

Acknowledgments. This work was supported by the University of Wollongong's URC small grants scheme. We would like to thank Rebecca Graham for her assistance in testing participants.

\section{References}

Agostinelli, G., Sherman, S., Fazio, R., \& Hearst, E. (1986). Detecting and identifying change: Additions versus deletions. Journal of Experimental Psychology: Human Perception and Performance, 12, 445-454.

Akhtar, N., \& Enns, J. T. (1989). Relations between convert orienting and filtering in the development of visual attention. Journal of Experimental Child Psychology, 48, 315-334.

Cole, G., Kentridge, R., Gellatly, A., \& Heywood, C. (2003). Detectability of onsets versus offsets in the change detection paradigm. Journal of Vision, 3(1):3, 22-31.

(4) 'Timed-out' trials accounted for $0.5 \%$ of the total number of experimental trials.

(5) 2 change types $\times 3$ cue types $\times 3$ number of parts changing; cell averages ranged 8.2 to 8.9. 
Favelle, S., Palmisano, S., Burke, D., \& Hayward, W. (2006). The role of attention in processing configural and shape information in 3-D novel objects. Visual Cognition, 13, 623-642.

Fernandez-Duque, D., \& Thornton, I. M. (2000). Change detection without explicit awareness: Do explicit reports underestimate the representation of change in the visual system? Visual Cognition, 7, 324-344.

Fernandez-Duque, D., \& Thornton, I. M. (2003). Explicit mechanisms do not account for implicit localization and identification of change: An empirical reply to Mitroff et al. (2002). Journal of Experimental Psychology: Human Perception and Performance, 29, 846-858.

Laloyaux, C., Destrebecqz, A., \& Cleeremans, A. (2006). Implicit change identification: A replication of Fernandez-Duque and Thornton (2003). Journal of Experimental Psychology: Human Perception and Performance, 32, 1366-1379.

Mitroff, S. R, Simons, D. J, \& Franconeri, S. L. (2002). The siren song of implicit change detection. Journal of Experimental Psychology: Human Perception and Performance, 28, 798-815.

Mondy, S., \& Coltheart, V. (2000). Detection and identification of change in naturalistic scenes. Visual Cognition, 7, 281-296.

Murphy, K., \& Andalis, J. (2013). Unconscious priming: Masked primes facilitate change detection and change identification performance. International Journal of Psychological Studies, 5, 45-54.

Rensink, R. A. (2002). Change detection. Annual Review of Psychology, 53, 245-277.

Rensink, R. A., O’Regan, J. K., \& Clark, J. J. (1997). To see or not to see: The need for attention to perceive changes in scenes. Psychological Science, 8, 368-373.

Scholl, B. J. (2000). Attenuated change blindness for exogenously attended items in a flicker paradigm. Visual Cognition, 7, 377-396.

Simons, D. J. (1996). In sight, out of mind: When object representations fail. Psychological Science, 7, 301-305.

Simons, D. J., \& Rensink, R. A. (2005). Change blindness: Past, present, and future. Trends in Cognitive Sciences, 9, 16-20.

Smilek, D., Eastwood, J. D., \& Merikle, P. (2000). Does unattended information facilitate change detection? Journal of Experimental Psychology: Human Perception and Performance, 26, 480-487.

Stolz, J., \& Jolicoeur, P. (2004). Changing features do not guide attention in change detection: Evidence from a spatial cuing paradigm. Psychonomic Bulletin \& Review, 11, 870-875.

Townsend, J. T., \& Ashby, F. G. (1983). The stochastic modeling of elementary psychological processes. Cambridge: Cambridge University Press.

Williams, P., \& Simons, D. J. (2000). Detecting changes in novel, complex three-dimensional objects. Visual Cognition, 7, 297-322. 